\title{
Lichen planus arising through the Koebner phenomenon in areas of Hijama
}

\section{Nabeel K. Al Hamzawi}

Department of Dermatology, Diwaniyah teaching hospital, Diwaniyah, Iraq

Corresponding author: Dr. Nabeel K.Al Hamzawi, E-mail: alhamzawi_n@yahoo.com

The Koebner phenomenon is the appearance of isomorphic pathologic lesions in traumatized but otherwise normal skin of patients who have cutaneous disorders. These new lesions are clinically and histologically identical to those in the diseased skin. Psoriasis, lichen planus, and vitiligo appear to display the true type of isomorphic response of Koenbner [1]. Herein, I describe a case of lichen planus arising through the Koebner phenomenon in the areas traumatized by Hijama.

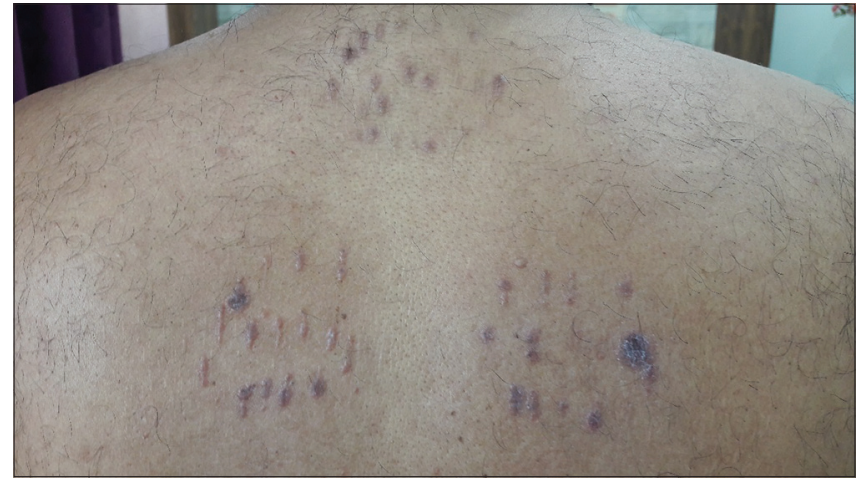

Figure 1: Three groups of violaceous flat-topped papules arising in areas of Hijama on the upper back, some of them have a linear shape and configuration.

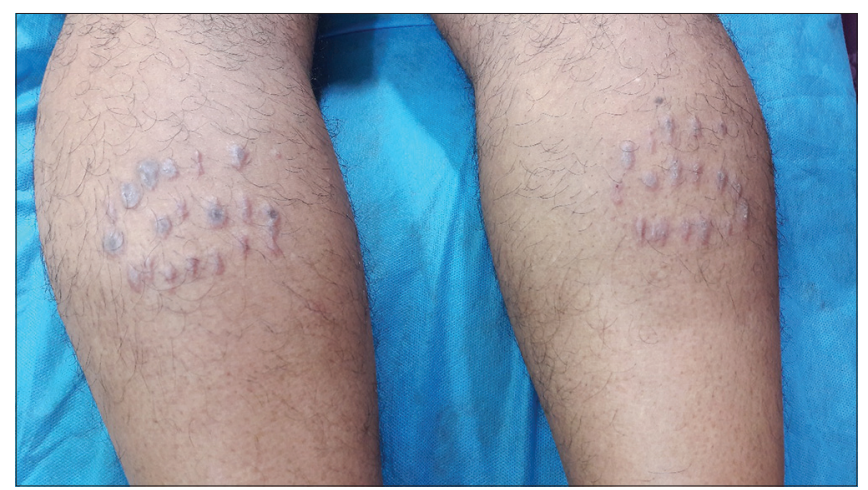

Figure 2: Groups of pruritic violaceous papules presenting on the back of the legs that traumatized by Hijama.
A 43-year-old man presented with multiple pruritic violaceous papules, arranged in groups, some of them have a linear shape and configuration. The lesions were confined to the upper back and the calf areas of the legs (Figs. 1 and 2). The patient's history showed that he was exposed to Hijama (cupping therapy) in these areas a month ago as a modality to treat the fibromyalgia that he was suffering from. A general examination revealed that he had similar previous lesions on the forehead and external genitalia (Figs. 3 and 4). The

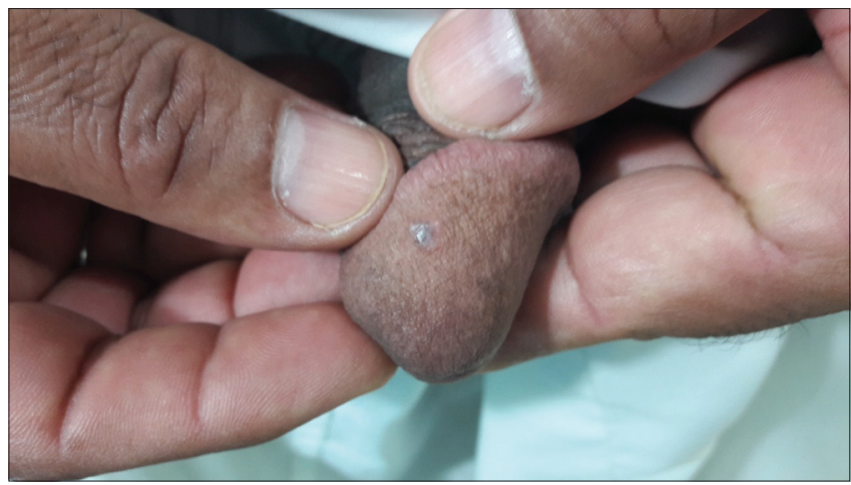

Figure 3: A violaceous plane papule of lichen planus on the glance penis.

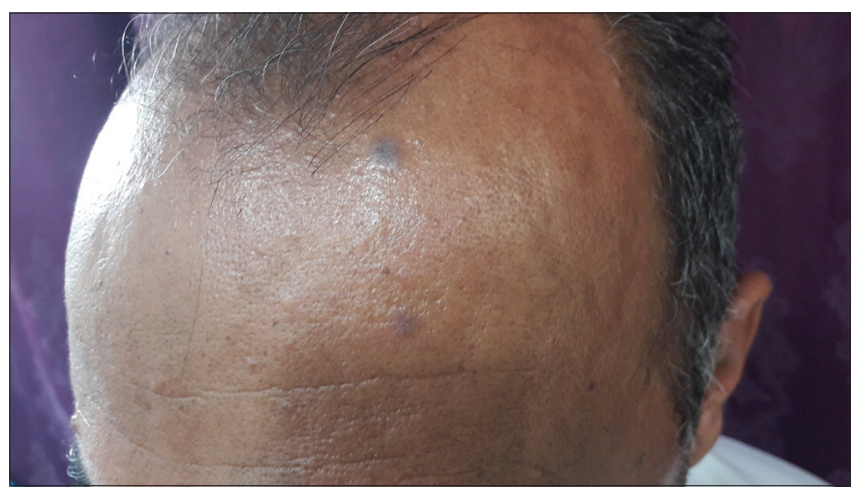

Figure 4: Lichen planus on the forehead of a 43-year-old man. 
histopathologic study was consistent with lichen planus. The patient has prescribed topical and systemic corticosteroid and antihistamine, with a good clinical response.

Hijama or cupping therapy is a form of alternative medicine in which a vacuum is created in a cup and placed on the skin. The therapist then removes the cup and uses a small scalpel to make a tiny cut on the skin surface. The procedure is used to treat anxiety, depression, back pain, fibromyalgia and high blood pressure. The local adverse effects of cupping include bruises, burn and infection. There were no clear contraindications to it apart from people with health problems due to side effects [2,3]. This case has added further contraindication to the use of Hijama in patients who have dermatoses provoked by trauma, such as psoriasis, lichen planus, and vitiligo.

\section{Consent}

The examination of the patient was conducted according to the Declaration of Helsinki principles.

\section{REFERENCES}

1. Thappa DM. The isomorphic phenomenon of Koebner. Indian J Dermatol Venereol Leprol. 2004;70:187-9.

2. El Sayed SM, Mahmoud HS, Nabo MMH. Methods of wet cupping therapy (Al-Hijamah): In light of modern medicine and prophetic medicine. Altern Integ Med. 2013;2:111.

3. Cao H, Li X, Liu J. An updated review of the efficacy of cupping therapy. PLOS One. 2012;7:e31793.

Copyright by Nabeel K. Al Hamzawi. This is an open-access article distributed under the terms of the Creative Commons Attribution License,

which permits unrestricted use, distribution, and reproduction in any medium, provided the original author and source are credited.

Source of Support: Nil, Conflict of Interest: None declared. 